\title{
Considerations for developing functional food products for the older population
}

\author{
H. Cummins ${ }^{1,2}$, C.A. Corish ${ }^{2}$, H.M. Roche ${ }^{2}$ and S.N. McCarthy ${ }^{1}$ \\ ${ }^{1}$ Teagasc Food Research Centre, Ashtown, Dublin 15, Ireland and \\ ${ }^{2}$ School of Public Health, Physiotherapy and Sports Science, UCD Conway Institutel UCD Institute of Food \& Health, \\ Belfield, Dublin 4, Republic of Ireland
}

Population ageing is a global demographic trend. Consequently, the importance of maintaining good health in older people is increasingly recognised, with food and nutrition acknowledged as modifiable factors. For this population group, adequate protein intake is essential to maintain muscle mass and function; it may also slow the development of sarcopenia ${ }^{(1)}$. In addition, meeting calcium and vitamin D requirements is vital in order to achieve optimal bone health into older age ${ }^{(2)}$. Functional food products which consider the needs of older adults are common in Asia; few products are available on the European market. Such products, including meal replacements and white milk powders, frequently have a medicinal focus in terms of product type, packaging and design ${ }^{(3)}$. This study sought to investigate the attitudes, motives and behaviours of older Irish adults towards the usage of functional foods and whether functional foods have a role to play in addressing their age related health issues. A series of focus groups were undertaken, with consumers aged $65-84$ years, $(n=61)$ from varying socio-demographic backgrounds. Focus groups were recorded, transcribed and thematically analysed using NVivo 11 QSR International.

'Perceived Need' and 'Trust' were recognised as prominent themes influencing functional food choice. For many participants, they did not perceive the need to use functional foods as believed that they were consuming a healthy balanced diet and did not require additional benefits from functional foods. For those where were consuming functional foods, it was for a specific health purpose such as lowing their cholesterol by consuming the plant stanol products. These products were only consumed after recommendation by a trusted person such as GP or family and even then, they were not fully convinced of their beneficial effects The most familiar and commonly used functional foods were fortified milks and cholesterol-lowering spreads. Participants who consumed these perceived them as easy substitutes for their equivalent non-functional food. These participants also stated that it is important to check with health care professionals before considering consuming functional foods. A strong distrust of the food industry and manufacturers across all focus groups was apparent and hence would not be viewed as a trusted source in communicating the benefits of functional foods.

Opportunities exist to produce foods that are specifically designed to meet the needs of the older person such as increasing protein content or fortifying with essential micronutrients. However, the communication strategy developed to promote these foods needs to be clear, simple and emphasize the benefits of these foods. Functional food ingredients must be easily incorporated into, or substituted for existing products. They must target a currently unmet need for older consumers and this message must come from trusted sources such as GP and family, to increase the likelihood of success.

NUTRIMAL (Novel NUTRItional solutions to combat chronic MALnutrition in the elderly) is funded by the Irish Department of Agriculture, Food and the Marine under the Food Institutional Research Measure (FIRM 14/F/822).

1. Paddon-Jones D \& Rasmussen B (2009) Curr Opin Clin Nutr Metab Care 12, 86-90.

2. A Prentice (2004) Public Health Nutr 7, 227-243.

3. Leatherhead Food Research. Mintel Global New Product Database: Foods for the Elderly 2010-2014. Mintel Group Ltd. Surrey: 2015. 